\title{
UN CASO PARA LA PRESERVACIÓN Y UTILIZACIÓN DE PARIENTES SILVESTRES DE LOS CULTIVOS EN PAPA (Solanum tuberosum L.) ${ }^{1}$
}

\section{E.L. Camadro ${ }^{2}$}

Manuscrito recibido: 02/08/2014

Aceptado: 07/09/2014

Disponible en línea: Diciembre 2014

\section{Resumen}

Los parientes silvestres de los cultivos (Crop wild relatives=CWR) constituyen un recurso crítico para abordar las necesidades de seguridad alimentaria, debido a que proveen diversidad genética para el mejoramiento de los cultivos, conducente al incremento de la plasticidad y productividad de los sistemas agrícolas. Sin embargo, los fitomejoradores típicamente no han desarrollado estrategias sistemáticas o globales integrales para la caracterización y utilización de CWR en el mejoramiento de cultivares. La papa provee un excelente caso de estudio para la utilización de germoplasma de CWR a fin de abordar las necesidades de seguridad alimentaria global. La cooperación y colaboración internacionales son críticas para coleccionar, caracterizar y utilizar CWR en anticipación a las necesidades futuras de producción. Tanto la preservación ex situ como in situ de las especies silvestres de la papa son esenciales para asegurar un plan de conservación global abarcativo. Las prioridades tope incluyen un inventario coordinado de las tenencias de los bancos de genes, seguido de la re-colección de CWR, y nueva colección donde ${ }^{1}$ Traducción de: S.H. Jansky, H. Dempewolf, E.L. Camadro, R. Simon, E. Zimnoch-Guzowska, D.A. Bisognin, M. Bonierbale . 2013. A Case for Crop Wild Relative Preservation and Utilization in Potato (Solanum tuberosum L.). Crop Sci. 53 (3): 746-754. No. de licencia 3453170018617 , de fecha 20 de agosto de 2014

${ }^{2}$ Investigador EEA Balcarce, Instituto Nacional de Tecnología Agropecuaria (INTA) y Consejo Nacional de Investigaciones Científicas y Técnicas (CONICET); Profesor Facultad de Cs. Agrarias, Universidad Nacional de Mar del Plata (UNMdP) 
existan brechas. El acceso a la diversidad de CWR continuará siendo crítico en tanto los fitomejoradores enfrenten el desafío de desarrollar cultivares que se ajusten a los nuevos sistemas de producción, especialmente en respuesta al cambo global. Con el advenimiento de la era genómica, están emergiendo nuevas visiones sobre estrategias de utilización de germoplasma. En adición a cubrir las brechas en las colecciones, será importante expandir los esfuerzos para caracterizar y utilizar CWR. Se necesita una estrategia sistemática e integral para evaluar CWR en bancos de genes por los caracteres necesarios para continuar con el progreso en el mejoramiento genético.

Palabras claves adicionales: parientes silvestres de los cultivos, mejoramiento de germoplasma, banco de genes, mejoramiento genético de cultivos

\section{A CASE FOR THE CONSERVATION AND UTILIZATION OF WILD RELATIVES IN CROP POTATOES (Solanum tuberosum L.)}

\section{Summary}

Crop wild relatives (CWR) offer a critical resource to address food security needs by providing genetic diversity for crop improvement, leading to increased plasticity and productivity of farming systems. However, plant breeders typically have not developed systematic or comprehensive strategies for the characterization and utilization of CWR for cultivar improvement. Potato provides an excellent case study for the utilization of CWR germplasm in addressing global food security needs. International cooperation and collaboration are critical in order to collect, characterize, and utilize CWR in anticipation of future production needs. Both 
ex situ and in situ preservation of wild potato species are essential to assure a comprehensive conservation plan. Top priorities include a coordinated inventory of gene bank holdings, followed by re-collection of CWR, and new collection where gaps exist. Access to CWR genetic diversity will continue to be critical as breeders face the challenge of developing cultivars that fit into new production systems, especially in response to climate change. With the advent of the genomics era, new visions of germplasm utilization strategies are emerging. In addition to filling gaps in collections, it will be important to expand efforts to characterize and utilize potato CWR. A systematic and integrated strategy is needed to evaluate CWR in gene banks for traits in order to continue breeding progress.

Additional Keywords: crop wild relatives, germplasm enhancement, gene bank, crop breeding.

\section{Introducción}

\section{El problema}

La degradación ambiental y el cambo climático plantean una amenaza a la seguridad alimentaria mundial. En décadas recientes han habido fallas en los cultivos, escasez de alimentos, reducciones en la productividad de cultivos, incrementos en el precio de los alimentos, y crisis económicas, poniendo de relieve la vulnerabilidad de los sistemas globales de producción de alimentos para sostener una población humana en constante crecimiento (Miller et al., 2010). Se estima que $20 \%$ las especies de plantas están en peligro de extinción en el futuro cercano (Brummitt y Bachman, 2010). Este número probablemente se incremente como resultado del 
cambio climático y la expansión de la urbanización y la producción agrícola. En adición, se espera que el rango geográfico de muchas especies de plantas se reduzca a la mitad en los próximos 50 años (Jarvis et al., 2008). Ya ha sido documentado que los cambios en el rango geográfico de muchas especies han ocurrido a tasas dos o tres veces mayores de lo que se pensó previamente (Chen et al., 2011).

Los parientes silvestres de los cultivos (CWR, por el inglés Crop Wild Relatives) ofrecen un recurso crítico para abordar las necesidades de seguridad alimentaria al proveer diversidad genética para el mejoramiento de los cultivos, lo que conduce al incremento de la plasticidad, sustentabilidad, y productividad de los sistemas de producción (Maxted et al.,2008). Históricamente ha habido indiferencia hacia los CWR tanto por parte de los científicos agrícolas, que creen que son demasiado silvestres para ser útiles, como por los científicos conservacionistas, que con frecuencia se focalizan en plantas raras sin consideración por el valor económico potencial. La importancia del recurso de CWR se está volviendo más evidente para los responsables de establecer políticas. En la Conferencia de las Naciones Unidas por el Cambio Climático en 2009, se enfatizó la necesidad de atención hacia la conservación y uso de la diversidad genética de los cultivos. Sin embargo, típicamente los fitomejoradores no han desarrollado estrategias sistemáticas o integrales para la caracterización y utilización de CWR en el mejoramiento de cultivares (Maxted et al., 2012). Consecuentemente, se requiere el desarrollo de estrategias viables para la conservación y uso sustentable de CWR para el mejoramiento de germoplasma de plantas cultivadas. En febrero de 2012, Global Crop Diversity Trust concertó una reunión de un grupo de expertos en CWR de papa para discutir estrategias para la colección y utilización de germoplasma. La presente revisión es un producto de esa reunión. La misma se desarrolló para describir la situación actual del recurso de germoplasma de papa y proveer un plan para su preservación y utilización en el futuro. 


\section{La situación actual}

La papa provee un excelente caso de estudio de la importancia de la utilización de germoplasma de CWR para abordar las necesidades de seguridad alimentaria global. Es el tercer cultivo alimentario más importante a nivel mundial, detrás del arroz y el trigo. En 2010, la producción mundial de papa fue 324 millones de toneladas, con un valor de cerca de U\$44.000 millones (FAO). En adición, en las décadas recientes la producción de papa se ha incrementado a un ritmo constante en los países en desarrollo debido a su alto rendimiento de alimento nutritivo. Por ejemplo, China es el productor mundial más grande de papa y la producción se está expandiendo para aumentar la estabilidad de los alimentos (Jansky et al., 2009a; Scott y Suárez, 2012). Los CWR de papa son abundantes y la mayoría de ellos pueden ser hibridados con la papa cultivada, ya sea directamente o aplicando estrategias que permiten sortear las barreras a la hibridación (Hanneman Jr., 1989; Camadro, 2010). De hecho, los CWR de papa han hecho importantes contribuciones a la resistencia a enfermedades, aumento de rendimiento y calidad mejorada a través del mejoramiento genético por más de 150 años (Hawkes, 1945, 1958; Rieman et al., 1954; Rudorf, 1958; Ross, 1966, 1979; Plaisted y Hoopes, 1989; Bradshaw y Ramsay, 2005; Bradshaw, 2009). Consecuentemente, se reconoce a la papa como un cultivo para el cual los CWR han sido prominentemente usados (Maxted et al., 2012). La Tabla 1 ilustra unas pocas contribuciones destacadas de los CWR a cultivares mejorados. Uno de los primeros ejemplos es el uso de $S$. demissum como fuente de genes mayores de resistencia a tizón tardío (Ross, 1966). En tanto estos genes fueron inicialmente significativos en el control del tizón tardío, no fueron duraderos. En contraste, la resistencia por gen mayor para el virus Y de la papa de S. stoloniferum ha probado ser duradera y se encuentra en un número de cultivares europeos (Flis et al., 2005). 
Otro éxito notable del uso de CWR en el mejoramiento genético de la papa es la variedad peruana María Huanca, que tiene un pedigrí complejo que contiene S. vernei (Llontop et al., 1989). Este cultivar es altamente resistente a dos razas del nematode del quiste blanco Globodera pallida, el virus X de la papa y el virus Y de la papa. El ejemplo final es el cultivar Lenape, que contiene $S$. chacoense (Akeley et al., 1968). Lenape está en el pedigrí de muchos cultivares modernos para hojuelas fritas y se le da crédito por contribuir a avances mayores en el mejoramiento genético por calidad de hojuelas fritas hacia fines del siglo XX (Love et al., 1998). Desafortunadamente, luego de su liberación, Lenape fue removida del mercado debido a niveles excesivos de glicoalcaloides en sus tubérculos, sin duda provenientes de $S$. chacoense, que es conocida por esta característica (Zitnak y Johnston, 1970). Este ejemplo ilustra la necesidad de que los programas de mejoramiento de germoplasma realicen evaluaciones integrales de sus productos para evitar la inclusión de propiedades indeseables en eventuales variedades.

Tabla 1. Ejemplos de avances mayores en el desarrollo de cultivares de papa como resultado de introgresión de germoplasma de CWR

\begin{tabular}{|l|c|c|c|}
\hline \multicolumn{1}{|c|}{ CONTRIBUCIÓN } & CLON & $\begin{array}{c}\text { DONANTE } \\
\text { CWR }\end{array}$ & REFERENCIA \\
\hline Resistencia a tizón tardío & Razas W & S. demissum & $\begin{array}{c}\text { Hawkes, 1945,1958; } \\
\text { Plaisted y Hoopes, 1989; } \\
\text { Bradshaw, 2009 }\end{array}$ \\
\hline $\begin{array}{l}\text { Resistencia a virus Y } \\
\text { de la papa }\end{array}$ & MPI 61.303/34 & S. stoloniferum & Bradshaw et al., 2006 \\
\hline Resistencia a nematodes & María Huanca & S. vernei & $\begin{array}{c}\text { Llontop et al., 1989; } \\
\text { Brodie et al., 1991 }\end{array}$ \\
\hline Calidad de procesamiento & Lenape & S. chacoense & Love et al., 1998 \\
\hline
\end{tabular}


Durante la domesticación, las poblaciones de plantas experimentan típicamente un severo cuello de botella genético (Feuillet et al., 2008). Este así llamado cuello de botella de la domesticación deja atrás mucha de la diversidad genética útil que podría contribuir al mejoramiento de los cultivos. La papa provee una buena ilustración de las consecuencias de los cuellos de botella genéticos. La papa cultivada se originó en el sur de Perú hace cerca de 10.000 años y luego se diseminó tanto en el norte como en el sur, manteniendo interacciones con sus parientes silvestres (Spooner et al., 2005). Sin embargo, hoy en día la mayor parte de la producción comercial de papa está concentrada en las regiones templadas de América del Norte, Europa, y Asia. Estas papas se originaron principalmente a partir de un conjunto pequeño de clones llevados a Europa en la segunda mitad del siglo XVI (Ames y Spooner, 2008). Presumiblemente, muchos de los clones no sobrevivieron el pasaje de Suramérica a Europa y otros que arribaron a Europa se perdieron debido a una pobre adaptación y presión por enfermedades. Por más de 300 años, las papas cultivadas en esas regiones tuvieron poco o ningún influjo de nueva diversidad genética. Las hambrunas de papa de medidos del siglo XIX en Europa fueron el resultado de la susceptibilidad genética de los cultivares a Phytopthora infestans, el agente causal del tizón tardío. El tremendo costo en vidas humanas a raíz de estas falencias en el cultivo de papa dieron ímpetu a los fitomejoradores para buscar nuevo germoplasma en un intento por producir cultivares menos susceptibles a enfermedades (Ross, 1966). Desde mediados del siglo XIX, los mejoradores de papa han estado experimentando con la introducción de germoplasma de CWR en sus programas, con grados de éxito variables. No obstante, la diversidad genética dentro y entre cultivares de papa de la zona templada norte sigue siendo bajo (Mendoza y Haynes, 1974; Wang, 2011). En tanto que la introgresión de genes específicos de las especies silvestres ha tenido un impacto significativo en el desarrollo de cultivares, sólo unas pocas especies han sido usadas ampliamente (Bradshaw et al., 2006). La mayoría de los programas de mejoramiento no han 
desarrollado una estrategia sistemática para incorporar ampliamente CWR en el germoplasma avanzado.

Las estimaciones del número de especies en grupos taxonómicos que contienen especies tuberosas de Solanum (sección Petota) han variado considerablemente en el tiempo y de acuerdo al taxónomo (Ovchinnikova et al., 2011). El número ha sido reducido en años recientes y actualmente se considera que la sección Petota incluye cuatro especies cultivadas (Spooner et al. 2007) y aproximadamente 110 especies silvestres tuberosas de Solanum (Spooner, 2009). Estas especies se distribuyen entre 16 países desde Estados Unidos a través de América Central y Suramérica hasta Chile y Argentina (Spooner y Salas, 2006). Las papas silvestres crecen desde el nivel del mar hasta los 4.300 m de altura, pero se encuentran más frecuentemente en altitudes de 2.000 a 4.000 m. Los CWR de la papa están adaptados a una variedad de ambientes, que incluye fríos pastizales de altura de los Andes, cálidos semidesiertos y hábitats secos estacionales, bosques lluviosos subtropicales húmedos y templados, campos cultivados $\mathrm{y}$, como epífitas, los árboles (Hawkes, 1990; Ochoa, 1990). Aproximadamente 70\% de las papas silvestres emparentadas son diploides. Los cultivares de las mayores regiones productoras del mundo son típicamente tetraploides, en tanto que las razas locales que aún se cultivan como alimentos básicos en Suramérica pueden ser diploides, triploides, tetraploides o pentaploides (Ochoa, 1990).

Los ecosistemas en los que crecen CWR se están tornando inestables debido al cambio climático, prácticas deficientes de manejo de la tierra, urbanización, y expansión de la infraestructura como desarrollo de carreteras (Maxted et al., 2012). Para papa, los escenarios de cambio climático predicen que el incremento en las temperaturas y la disminución de la disponibilidad de agua darán como resultado una reducción sustancial del rendimiento en el nivel mundial de hasta $32 \%$ en 2050 (Schafleitner et al., 2011). Varias amenazas en los Andes y México, donde se encuentran 
la mayoría de las especies silvestres de papa, incluyen la minería, el sobrepastoreo, la expansión de animales domésticos exógenos (como cerdos y cabras), la deforestación, la expansión de la agricultura y la pérdida de hábitats en general. Las regiones bajo la mayor amenaza para los cultivos y sus parientes silvestres incluyen las zonas altas tropicales de Suramérica, Asia y África, y partes del sur de África.

Los CWR son con frecuencia clasificados de acuerdo al concepto de pozo génico desarrollado por Harlan y de Wet (1971). Los pozos génicos de los cultivos prioritarios para conservación de CWR son típicamente GP1B (parientes silvestres que se cruzan directamente con los cultivares) y GP2 (parientes silvestres que se cruzan con algunas manipulaciones) (Maxted et al., 2012). Estos recursos de germoplasma son los que con mayor probabilidad serán introgresados en los cultivares a través de métodos convencionales de mejoramiento genético. Las especies silvestres y cultivadas de papa se encuentran en una serie de ploidía que va de diploide a hexaploide. Un determinante del éxito de la hibridación interespecífica en papa es el número de balance del endosperma (NBE) (Johnston et al., 1980). Las especies con valores NBE que concuerdan, sin importar la ploidía, se hibridarán siempre y cuando estén ausentes otras barreras. Los valores de ploidía/NBE de los parientes silvestres de la papa incluyen 6x (4NBE), 4x (4NBE), 4x (2NBE), 2x (2 NBE), y 2x (1NBE). La aplicación del concepto de pozo génico a la papa colocaría a las especies con 6x (4NBE), 4x (4NBE), 4x (2 NBE), y 2x (2NBE) en GP1B y a las especies $2 x$ (1NBE) en GP2. Hay dos especies de Solanum que no son tuberosas, que serían GP3 (parientes silvestres que no se cruzan directamente con la papa cultivada). Dado que no se pueden hibridar con la papa, no se les ha asignado un valor de NBE. Los valores de ploidía y NBE de las especies silvestres de Solanum han sido provistos por Spooner y Hijmans (2001). En adición al NBE, otras numerosas barreras pre- y post-cigóticas a la hibridación son comunes en cruzamientos interespecíficos (Fritz y Hanneman Jr., 1989; Camadro et al. 1998, 2004, 2012; Jackson y 
Hanneman Jr. 1999; Chen et al. 2004; Weber et al., 2012). Estas barreras confunden los intentos para aplicar el concepto de pozo génico a las papas.

Muchos de los parientes silvestres y cultivados de la papa están representados en bancos de genes a través del mundo. Estas facilidades proveen acceso a CWR para actividades de investigación y mejoramiento genético. Los bancos de genes de papa incluyen el Centro Internacional de la Papa (CIP, Lima, Perú), el Proyecto de Introducción de Papa de Estados Unidos (NRSP-6, Sturgeon Bay, Wisconsin, USA), la Colección de Papa Holandesa-Alemana (CGN, Wageningen, Países Bajos, y BGCR, Braunschweig, Alemania), el Instituto de Investigación en Genética de Plantas y Plantas Cultivadas (GLKS, Groß Lüsewitz, Alemania), la Colección de Papa de la Comunidad Británica de Naciones (CPC, Dundee, Scotland), el Instituto N.I. Vavilov (VIR, San Petesburgo, Rusia), el Instituto Nacional de Tecnología Agropecuaria (INTA, Balcarce, Argentina), y Cenargen (EMBRAPA, Brasilia, Brasil). Una base de datos inter-bancos de genes contiene registros de siete de esos bancos de genes (Huamán et al., 2000). El CIP actúa como servidor anfitrión de una base de datos inter-bancos de genes, que puede ser encontrada en línea en http://germplasmdb.cip.cgiar.org. Sin embargo, no es usualmente actualizada en forma regular. La falta de financiación estable para el mantenimiento de bases de datos científicos es un problema serio (Baker, 2012). Global Crop Diversity Trust ha asumido, sin embargo, el compromiso de respaldar en años múltiples la base de datos Genesys (http://genesys-pgr.org/). Este sistema se focaliza en coleccionar datos a nivel de introducción de bancos de genes del mundo entero.

\section{Llamado a la Acción}


La cooperación y colaboración internacionales son críticas para coleccionar, caracterizar y utilizar CWR en anticipación a las futuras necesidades de la producción de papa. Primero, se debe hacer un inventario de CWR existentes en sus ambientes naturales y en bancos de genes. Sobre la base de niveles de amenaza al hábitat bien definidos, endemismo, rango de distribución, datos de caracterización, esfuerzos de conservación, y valor de uso, entre otros factores, se debería promover un enfoque sistemático de conservación in situ en centros de diversidad de CWR. En adición, se deberían desarrollar prioridades de colección y preservación. Las colecciones altamente prioritarias deberían ser aquellas en los sitios amenazados y para especies que están sub-representadas en bancos de genes. Al mismo tiempo, se deberían hacer esfuerzos sistemáticos e integrales para desarrollar e implementar las estrategias más efectivas para caracterización, documentación, e incorporación de gemoplasma de CWR en poblaciones de mejoramiento para el desarrollo de cultivares de papa.

Las tecnologías de sistemas de información geográfica han permitido una mejor comprensión de las distribuciones de especies de papa sobre la base de datos de pasaporte de las colecciones de bancos de genes (Hijmans y Spooner, 2001; Hijmans et al., 2002). Sin embargo, en décadas recientes, no se han realizado investigaciones a nivel de campo sobre cambios de hábitat y estado de conservación in situ. Nuestra comprensión de la ecología y dinámica de poblaciones de los parientes silvestres de la papa es generalmente muy pobre. Se han documentado varios sesgos en las colecciones de bancos de genes que deben ser consideradas en esfuerzos por cubrir las brechas en los recursos in situ de germoplasma (Hijmans et al., 2012).

Un análisis de brecha ayudará a determinar cuán ampliamente se encuentran reunidos y conservados los recursos CWR en bancos de genes. Este análisis compara el rango natural de CWR con aquel documentado en inventarios de banco de genes. Los resultados del análisis de brecha proveen dirección para los esfuerzos de expansión de colecciones de CWR que están sub- 
representadas ex situ en bancos de genes (Maxted et al. 2008; Ramírez-Villegas et al., 2010). Un equipo en el Centro Internacional de Agricultura Tropical (CIAT, Cali, Colombia) está trabajando en el análisis de brecha para CWR de papa, en coordinación con CIP y Global Crop Diversity Trust. Cuando estén disponibles, los resultados de este proyecto serán expuestos en: http://www.cwrdiversity.org/conservation-gaps/. Se deberían también incluir en el análisis, si se dispone de datos, criterios prioritarios adicionales tales como amenazas a las poblaciones silvestres (por ej., minería, urbanización, sobrepastoreo, cambio climático), y grado de parentesco de taxa con las especies cultivadas. Para incluir una imagen más completa, el método debería idealmente ir unido a la valoración del estado de conservación percibido, por ejemplo, en parques y otras áreas protegidas.

En adición a las determinadas por el análisis de brecha, las prioridades de colección pueden enfocarse en especies GP1 y GP2, que son las qué más fácilmente pueden ser introgresadas en la papa cultivada. Sin embargo, la mayoría de los CWR de la papa entran en esta categoría, de modo que se necesitan criterios complementarios adicionales si se han de establecer prioridades. Alternativamente, un argumento que puede darse es que las especies GP3 deberían recibir prioridad, porque tienen el potencial para contribuir genes únicos para caracteres que no son actualmente considerados como importantes por los fitomejoradores, pero que pueden serlo en el futuro. Por ejemplo, se han encontrado propiedades novedosas de fitonutriente y almidón en especies GP3 (Navarra et al. 2011; Fajardo y Jansky, 2012). Entre los taxa prioritarios, aquellos con necesidad más urgente de conservación tienen típicamente un rango geográfico limitado (Maxted et al., 2012). En tanto que las expediciones de colección deberían focalizarse en añadir a la diversidad genética que ya se encuentra en las colecciones actuales, la re-colección de poblaciones que se mantienen en bancos de genes proveerían una oportunidad para evaluar la erosión genética en el campo y la deriva génica en colecciones de bancos de genes. Son 
recomendables los estudios de campo y de laboratorio, incluyendo análisis genéticos y de poblaciones, para guiar las estrategias de colección y conservación de germoplasma, y para informar a los mejoradores sobre enfoques efectivos para el mejoramiento del cultivo (del Río et al., 1997; Del Río y Bamberg, 2003; Bamberg y del Río, 2006; Bamberg et al., 2009; Camadro, 2012).

Un desafío considerable que se presenta para la colección de parientes silvestres de la papa es que con frecuencia se encuentran en asociaciones simpátricas en las que pueden fácilmente hibridarse. Como resultado, los límites entre especies pueden fácilmente volverse borrosos (Masuelli et al., 2009; Camadro et al., 2012). La segregación transgresiva en estas poblaciones híbridas puede permitirles sobrevivir in hábitats que son más extremos que los de cualquiera de sus padres. Es entonces importante incluir en la colecciones los híbridos que se encuentren naturalmente, pero mantenerlos separados $\mathrm{y}$, cuando sea posible, claramente etiquetarlos como tales. También son críticos la información descriptiva del hábitat, la distribución espacial, ecología, geografía y entorno, tales como amenazas y esfuerzo de conservación. La evolución en marcha, mediada por el flujo génico entre especies cultivadas y silvestres, ocurre en el centro de origen de los Andes y debería ser documentada más detalladamente (Celis et al., 2004; Scurrah et al., 2008). Poco se sabe de lo que sucede luego de que haya ocurrido el flujo génico entre parientes silvestres y cultivados en escenarios agrícolas. La descendencia debe pasar por una serie de pasos críticos de selección natural y humana para convertirse en cultivares y razas locales viables, Por cierto, pueden aprenderse lecciones del estudio de casos exitosos de "premejoramiento" espontáneo mediado por agricultores, por selección que da por resultado el influjo de genes de especies silvestre en el pozo génico cultivado (Brush et al., 1981).

Sería útil tener una estrategia sistemática para caracterizar el valor de la variación fenotípica de CWR para el mejoramiento del cultivo. En 1920, el científico ruso Nikolai Vavilov propuso la 
Ley de las Series Homólogas en la Variación para explicar la significación de la diversidad genética en parientes de los cultivos (Vavilov, 1922). Básicamente, propuso que el conocimiento de caracteres en una especie puede ser usado para predecir la presencia de caracteres similares en especies emparentadas. Este concepto es útil para algunos caracteres y algunas especies de papa (Jackson, 1990). Una serie de estudios recientes ha intentado predecir la distribución de fenotipos útiles en los parientes silvestres de la papa basados en taxonomía y datos biogeográficos (Jansky et al., 2006, 2008, 2009b; Spooner et al., 2009, Cheng et al., 2010; Cai et al., 2011). Sin embargo, el éxito de la predicción ha sido como mucho débil. Específicamente, los datos biogeográficos para papa no han tenido valor o el valor ha sido muy bajo para predecir niveles de resistencia debido a la alta variabilidad intra-introduccción (por ej., Jackson, 1990; Jansky, 2008). Esto implica que probablemente se pueda inferir una mayor variabilidad genética de la papa a partir de la distribución geográfica, pero posiblemente también que los predictores biogeográficos usados pueden no capturar adecuadamente las características de los microhábitats. En conclusión, los análisis biogeográficos para predecir caracteres necesitan ser manejados sobre la base de caso por caso y deberían ser complementados con datos genéticos o genómicos de alta resolución.

Como se indica previamente, una importante limitación de los análisis predictivos es que los datos de pasaporte de expediciones de colección son dispersos. En consecuencia, no se pueden elucidar los estreses ambientales locales y hábitats. En el futuro, deberían desarrollarse protocolos de colección estandarizados y abarcativos que incluyan información precisa de la localidad de colección, incluyendo tipo de suelo, presencia de enfermedades y plagas, tamaño de la población, diversidad de especies, disturbios del hábitat, y localización relativa de la actividad agrícola.

Un elemento importante de los datos de pasaporte es la información precisa de latitud y longitud, preferentemente usando un dispositivo de sistema de posicionamiento global (GPS). Esto permite 
que los sistemas geográficos informáticos complementen datos adicionales, tales como datos climáticos de bases de datos globales espaciales, e inferir índices biogeográficos y de vegetación que usualmente no son medibles durante la visita de colección. Aunque los conjuntos de datos espaciales no son tan precisos como los datos del hábitat observados in situ, los datos darán sustento para tres usos principales: a) expandir la caracterización ambiental local, b) detectar patrones en escala amplia de diversidad genética y c) identificar sitios con hábitats similares, con el potencial de orientar colecciones adicionales que apunten a cubrir las brechas.

La estructura genética de poblaciones y su distribución espacial deben ser tenidas en cuenta durante la colección. Es importante registrar el número de individuos muestreados para subsiguientemente estar en condiciones de estimar frecuencias génicas en introducciones individuales. Idealmente, el coleccionista emplearía diferentes estrategias de muestreo dependiendo del sistema de reproducción y la distribución del taxón. Especialmente en la era genómica, los científicos pueden comenzar a tratar cada introducción de un banco de genes como una colección de genes y por tanto combinar individuos para esfuerzos en genética y mejoramiento genético (Maxted et al. 2012).

En adición a cubrir las brechas en las colecciones, será importante expandir los esfuerzos para caracterizar y utilizar CWR. Proponemos un enfoque por tres flancos para desarrollar sistemáticamente una estrategia. Primero, en el corto plazo deberían desarrollarse poblaciones diploides y compartirse entre fitomejoradores que se focalizan en el mejoramiento de germoplasma. Segundo, la poliploidización sexual sería utilizada para crear clones tetraploides que combinen caracteres de ambos padres. Finalmente se lanzaría una red internacional de evaluación multi-sitio para materiales pre-mejorados. En este paso final se aplicaría preferentemente un enfoque de selección amplia del genoma y se implementaría conjuntamente un eje centralizado de alto caudal de procesamiento para fenotipificación cuantitativa usando 
ambientes controlados cuando fuera posible. El mejoramiento de germoplasma, a diferencia del mejoramiento genético varietal, debería enfocarse en nuevos productos intermedios en lugar de productos finales de variedades mejoradas. Se requiere atención para reconocer fenotipos "inesperados" valiosos, en tanto que los caracteres blanco se ensamblan a partir de combinaciones de CWR. Será también importante incorporar mecanismos que facilitarán el uso posterior del germoplasma diploide. Por ejemplo, como se mencionó anteriormente, la producción de gametos $2 n$ permitiría la poliploidización sexual de germoplasma diploide seleccionado (den Nijs y Peloquin, 1977). La explotación de este mecanismo permitirá la transferencia directa de caracteres deseables de CWR diploides a germoplasma cultivado tetraploide (Herriott et al., 1990; Hayes y Thill, 2002; Bisognin et al., 2005; Frost et al., 2006).

La comunidad de investigación en papa no se ha focalizado en maximizar los efectos de la segregación transgresiva o heterosis a través de la generación de un conjunto estratégico de diversos híbridos cultivar-especie silvestre. En trigo, se han creado nuevas sintéticas en gran escala sobre la base de novedosas combinaciones de CWR y luego han sido seleccionadas por tolerancias a estreses bióticos y abióticos (Mujeeb-Kazi et al., 2008). Este germoplasma ha provisto un recurso a nivel mundial para el mejoramiento genético de trigo e ilustra el valor de la introgresión de germoplasma de CWR en líneas adaptadas de mejoramiento. Una estrategia similar podría ser usada en papa para desarrollar poblaciones a partir de las cuales seleccionar poblaciones pre-mejoradas para caracteres múltiples y clones para mejoramiento genético.

El tercer flanco en la estrategia de utilización de CWR es el desarrollo de una red de trabajo para observación de caracteres, similar en concepto a la red de trabajo GEO-BON focalizada en la colección a largo plazo y curación de datos ecológicos (Scholes et al., 2012). Esta facilidad reuniría los datos de evaluación existentes de los colaboradores a nivel mundial y usaría esos 
conjuntos de datos para establecer una red de trabajo de sitios de ensayos que podrían ser usados por la comunidad para generar nuevos datos de evaluación sobre materiales pre-mejorados de papa en el largo plazo. El germoplasma incluido en tal red de trabajo de sitios de evaluación sería un conjunto que incluye híbridos cultivado x silvestres que son de interés para el contexto local de cada sitio de evaluación, pero que también serían de valor potencial en otras localidades. El conjunto de líneas pre-mejoradas podría incluir materiales pre-existentes así como algunos materiales que serían creados por investigadores en cada sitio usando una diversidad de introducciones de CWR y líneas receptoras de S. tuberosum con características útiles para cada programa. Los clones parentales receptores de S. tuberosum probablemente serían dihaploides o cultivares exitosos diploides (razas locales) que hayan sido cuidadosamente caracterizados con marcadores moleculares sólidos. Tal enfoque crearía conjuntos de materiales altamente diversos que podrían ser útiles para descubrir caracteres inesperados en fondos genéticos mejorados agronómicamente. Una vez que se seleccionen y se pongan a disposición, el uso de conjuntos de progenitores receptores diploides y tetraploides bien caracterizados facilitaría el monitoreo de genes introgresados y caracteres en representantes del pozo genético cultivado. Varios elementos de esta estrategia propuesta han sido llevados a cabo últimamente en centros de investigación en mejoramiento genético, con contribuciones promisorias notorias para los programas de desarrollo de cultivares en marcha en sus respectivos países (Zimnoch-Guzowska et al., 1998). Sin embargo, estos esfuerzos han sido llevados a cabo en una escala menor que la se propone aquí.

La clave del éxito de este enfoque será el uso de protocolos estandarizados para la evaluación de recursos genéticos y líneas de mejoramiento genético. Esto incluye el desarrollo de ontologías de carácter, diccionarios de carácter, y sistemas apropiados de manejo de datos a través de los cuales los datos resultantes de la evaluación pueden ser compartidos entre colaboradores a través de 
localidades y hacerse disponibles a una comunidad más amplia. Tales esfuerzos se están llevando a cabo en papa: (a) un conjunto de protocolos estandarizados se encuentra disponible en un libro de campo electrónico (Bonierbale et al., 2007), (b) se está desarrollando una ontología de papa (Shrestha et al., 2010) y (c) se están también desarrollando sistemas de datos que apuntan a manejar conjuntos de datos en gran escala (Guberman et al., 2011). Actualmente se están expandiendo los protocolos de la Guía Internacional de Cooperadores del CIP para incluir evaluación por tolerancia a sequía. El trabajo de ontología en papa se lleva a cabo como parte de una comunidad, en un esfuerzo por mantener la consistencia y la transferencia de conocimiento dentro y a través de los cultivos. El sistema de datos actualmente en uso en el CIP como plataforma de información sobre mejoramiento genético de papa, hace uso de una base de datos desarrollada para albergar datos de tamaño genómico para almacenar datos adicionales de fenotipicación y ambiente, facilitando la integración de datos. El desarrollo del protocolo estándar incluiría también el entrenamiento para llevar a cabo la fenotipificación en el campo con un programa asociado de aseguramiento de calidad para repetibilidad y reproducibilidad. Los conjuntos de datos resultantes se convertrían luego en un recursos comunitario y pueden ser "conservados" en perpetuidad, independientemente de los caminos que tomen las carreras de investigadores individuales. Otra base de datos relevante es la Red Genómica SOL (SGN), que contiene datos genéticos, genómicos y fenotípicos de las Solanaceae, incluyendo la papa (Bombarely et al., 2011). El objetivo de largo plazo de SGN es crear una red de recursos para enlazar genotipo con fenotipo de modo de proveer percepciones de la adaptación y diversificación de plantas.

\section{Conclusiones y el camino hacia delante}


Colección y preservación. La conservación y el uso sustentable de CWR se ha convertido en prioridad en la agenda internacional de conservación (Maxted et al., 2012). Vale la pena conservar CWR porque hacen una contribución significativa a los servicios del ecosistema (Ford-Lloyd et al., 2011). Tanto la preservación ex situ como in situ de las especies silvestres de papa es esencial para asegurar un plan de conservación abarcativo. Una prioridad tope es el reinventario de colecciones de bancos de germoplasma seguida de la re-colección de CWR cuando se detecten brechas. Lo que se deben conservar son frecuencias génicas de poblaciones naturales y no genotipos porque los genes, no los genotipos, se transmiten a través de generaciones. El acceso a la diversidad genética de CWR continuará siendo crítico en tanto los fitomejoradores enfrenten el desafío de desarrollar nuevos cultivares que se ajusten a nuevos sistemas de producción, especialmente en respuesta al cambio climático. Con el advenimiento de la era genómica, están emergiendo nuevas visiones sobre estrategias de utilización de germoplasma. Por ejemplo, los bancos de genes tienen el potencial para evolucionar desde colecciones de semillas hasta centros de investigación dinámicos para actividades de minería de genes (McCouch et al., 2012).

Evaluación y mejoramiento genético. Se necesita una estrategia para evaluar CWR en bancos de genes por los caracteres necesarios para continuar con el proceso de mejoramiento genético. Entre los derivados adaptados, probablemente sería productiva la caracterización por un rango de caracteres (Jansky y Rouse, 2003). En adición, la completa evaluación de poblaciones durante el curso del mejoramiento genético, como en programas de retrocruzamientos y de selección asistida por marcadores, debe ser una prioridad para poder encontrar lo inesperado mientras se evalúan las contribuciones de los CWR en fondos genéticos relevantes. Este programa debería dividirse entre centros de investigación bajo un paraguas global y requiere financiamiento conjunto durable. 


\section{Conflictos de interés}

La autora declara que no existen conflictos de interés.

\section{Agradecimientos}

Global Crop Diversity Trust agradece por reunirse en el taller de expertos en parientes silvestres del cultivo de papa a través de la iniciativa "Adaptando la agricultura al cambio climático: una iniciativa global para coleccionar, conservar y usar parientes silvestres", que es financiada por el gobierno de Noruega. Stef de Hann, Henry Juárez, Benny Ordoñez, Alberto Salas y Jenny van Beem del Centro Internacional de la Papa revisaron este manuscrito y realizaron valiosas contribuciones. También se agradecen las contribuciones de los siguientes participantes al taller: Walter Amoros, Mathew Andrew, Arturo Brenes, Paolo Donini, Tatiana Gravilenko, Wolfgang Grunenber, Kazuyoshi Hosaka, Andrew Jarvis, Sandra Knapp, Hannele Lindqvist-Kreuze, Elisa Mihovilovich, Philippe Monneveux, Flor Rodríguez, Manuel Ruiz, Tiina Sarkinnen, Rena Sanemoto, María Scurrah, Craig Yencho, y Maria Zaharieva.

\section{Literatura citada}

Akeley, R.V.; Mills W.R.; Cunningham, C.E; Watts, J. 1968. Lenape: A new potato variety high in solids and chipping quality. Am. Potato J. 45: 142-145.

Ames, M.; Spooner, D.M. 2008. DNA from herbarium specimens settles a controversy about origins of the European potato. Am. J. Bot. 95: 252-257.

Baker, M. 2012. Databases fight funding cuts. Nature 489: 19.

Bamberg, J.B.; del Río, A.H. 2006. Seedling transplant selection does not cause genetic shifts in genebank populations of inbred potato species. Crop Sci. 46: 424-427. 
Bamberg, J.; del Río, A.H.; Moreyra, R. 2009. Genetic consequences of clonal versus seed sampling in model populations of two wild potato species indigenous to the USA. Am. J. Potato Res. 86: 367-372.

Bisognin, D.A.; Douches, D.S. ; Buszka, L.; Bryan, G.; Wang, D. 2005. Mapping late blight resistance in Solanum microdontum Bitter. Crop Sci. 45: 340-345.

Bombarely, A., Menda, N.; Tecle, I.; Buels, R.; Strickler, S.; Fischer-York, T.; Pujar, A.; Leto, J.; Gosselin, J.; Mueller, L. 2011. The Sol Genomics Network (solgenomics.net): Growing tomatoes using Perl. Nucleic Acids Res. 39: D1149-55.

Bonierbale, M.; De Haan, S.; Forbes, G.A. 2007. Procedures for Standard Evaluation Trials of Advanced Potato Clones. An Intrenational Cooperators' Guide. International Potato Center, Lima, Perú.

Bradshaw, J.E. 2009. Potato breeding at the Scottish Plant Breeding Station and the Scottish Crop Research Institute: 1920-2008. Potato Res. 52: 141-172.

Bradshaw, J.E.; Bryan, G.J.; Ramsay, G. 2006. Genetic resources (including wild and cultivated Solanum species) and progress in their utilisation in potato breeding. Potato Res. 49: 49-65.

Bradshaw, J.E.; Ramsay, G. 2005. Utilisation of the Commonwealth Potato Collection in potato breeding. Euphytica 146: 9-19.

Brodie, B.; Plaisted, R.; Scurrah M. de. 1991. The incorporation of resistance to Globodera pallida into Solanum tuberosum germplasma adapted to North America. Am. Potato J. 68:1-12

Brummitt, N.; Bachman, S. 2010. Plants under pressure: A global assessment. The first report of the IUCN samples red list index for plants. Packer, L. (ed.). Natural History Museum, London, UK.

Brush, S.B.; Carney, H.J.; Huamán, Z. 1981. Dynamics of Andean potato agriculture. Econ. Bot. 35: 70-88. 
Cai, X.; Spooner D.; Jansky, S. 2011. A test of taxonomic and biogeographic predictivity: Resistance to potato virus Y in wild relatives of the cultivated potato. Phytopathology 101: 10741080.

Camadro, E.L. 2010. Characterization of the natural genetic diversity of Argentinean potato species and manipulations for its effective use in breeding. The Am. J. Plant Sci. Biotech. 3 (Special issue 1): 65-71.

Camadro, E.L. 2012. Relevance of the genetic structure of natural populations, sampling and classification approaches for conservation and use of wild crop relatives: potatoes as an example. Botany 90: 1065-1072.

Camadro, E.L.; Carputo, D.; Peloquin, S.J. 2004. Substitutes for genome differentiation in tuberbearing Solanum: interspecific pollen-pistil incompatibility, nuclear-cytoplasmic male sterility, and endosperm. Theor. Appl. Genet. 109: 1369-1376.

Camadro, E.L.; Erazzú, L.E.; Maune, J.F:, Bedogni, M.C. 2012. A genetic approach to the species problem in wild potato. Plant Biol. 14: 543-554.

Camadro, E.L.; Verde, L.A.; Marcellán, O.N. 1998. Pollen-pistil incompatibility in a diploid hybrid potato population with cultivated and wild germplasm. Am. J. Potato Res. 75: 81-85.

Celis, C.; Scurrah, M.; Cowgill, S.; Chumbiauca, S.; Green, J.; Franco, J.; Main, G.; Kiezebrink, D.; Visser, R.; Atkinson, H. 2004. Environmental biosafety and transgenic potato in a centre of diversity for this crop. Nature 432: 2-5.

Chen, I.-C.; Hill, J.K.; Ohlemüller, R.; Roy, D.B.; Thomas, C.D. 2011. Rapid range shifts of species associated with high levels of climate warming. Science 333: 1024-1026.

Chen, Q.; Lynch, D.; Platt, H.W.; Shi, H.Y.L.; Li, H.J.; Beasly, D.; Rakosy-Tican, L.; Theme, R. 2004. Interspecific crossability and cytogenetic analysis of sexual progenies of Mexican wild diploid 1EBN species Solanum pinnatisectum and S. cardiophyllum. Am. J. Potato Res. 81: 159169. 
Chung, Y.S.; Holmquist, K.; Spooner, D.M.; Jansky, S.H. 2010. A test of taxonomic and biogeographic predictivity: Resistance to soft rot in wild relatives of cultivated potato. Phytopathology 101: 205-212.

Del Río, A.H.; Bamberg, J.B. 2003. The effect of genebank seed increase on the genetics of recently collected potato (Solanum) germplasm. Am. J. Potato Res. 80: 215-218.

Del Río, A.H.; Bamberg, J.B.B.; Huamán, Z.; Salas, A.; Vega, S.E. 1997. Assessing changes in the genetic diversity of potato gene banks. 1 . Effects of seed increase. Theor. Appl. Genet. 95: 199-204.

Del Río, A.; Bamberg, J., Huamán, Z.; Salas, A.; Vega, S. 2001. Association of ecogeographical variables and RAPD marker variation in wild potato populations of the USA. Crop Sci. 41: 870878

Den Nijs, T.P.M.; Peloquin, S.J. 1977. 2n gametes in potato species and their function in sexual polyploidization. Euphytica 26: 585-600.

Fajardo, D.; Jansky, S.H. 2012. Studies of amylose content in potato starch. Am. J. Potato Res. 89: 35 (abstr.).

Feuillet, C.; Langridge, P.; Waugh, R. 2008. Cereal breeding takes a walk on the wild side. Trends Genet. 24: 24-32.

Flis, B.; J. Hennig, J.; D.S.-Zyta, C.; Gebhardt, C.; Marczewski, W. 2005. The Ry-fsto gene from Solanum stoloniferum for extreme resistant to Potato virus Y maps to chromosome XII and is diagnosed by PCR marker GP122 718 in PVY resistant potato cultivars. Mol. Breed. 15: 95-101.

Ford-Lloyd, B.V.; Schmidt, M.; Armstrong, S.J.; Barazani, O.; Engels, J.; Hadas, R.;. Hammer K.; Kell, S.P.;Kang, D.; Khoshbakht, K.; Li. Y.;Long, C.; Lu, B.-R.; Ma, K.; Nguyen, V.T.; Qiu, L.; Ge, S.; Wei, W.; Zhang, Z; Maxted, N. 2011. Crop wild relatives-Undervalued, underutilized and under threat? BioScience 61: 559-565. 
Fritz, N.K.; Hanneman Jr, R.E. 1989. Interspecific incompatibility due to stylar barriers in tuberbearing and closely related non-tuber-bearing Solanums. Sex. Plant Reprod. 2: 184-192.

Frost, K.E.; Jansky, S.H.; Rouse, D.I.. 2006. Transmission of Verticillium wilt resistance to tetraploid potato via unilateral sexual polyploidization. Euphytica 149: 281-287.

Guberman, J.M.; Ai, J.; Arnaiz, O.; Baran, J.; Blake, A. et al. 2011. BioMart Central Portal: an open database network for the biological community. Database $\square$ : the journal of biological databases and curation 2011: bar041.

Hanneman Jr., R.E. 1989. The potato germplasm resource. Am. Potato J. 66: 655-667.

Harlan, J.; De Wet, J. 1971. Toward a rational classification of cultivated plants. Taxon 20: 509_ 517.

Hawkes, J. 1945. The indigenous American potatoes and their value in plant breeding. Empire J. Exp. Agric.: 11-40.

Hawkes, J.G. 1958. Significance of wild species and primitive forms for potato breeding. Euphytica 7: 257-270.

Hawkes, J.G. 1990. The Potato: Evolution, Biodiversity, and Genetic Resources. Smithsonian Institution Press, Washington, D. C.

Hayes, R.J.; Thill, C.A.. 2002. Introgression of cold (4 C) chipping from 2x (2 Endosperm Balance Number) potato species into $4 \mathrm{x}(4 \mathrm{EBN})$ cultivated potato using sexual polyploidization. Am. J. Potato Res. 79: 421-431.

Herriott, A.B.; Haynes Jr., F.L.; Shoemaker, P.B.. 1990. Inheritance of resistance to early blight disease in tetraploid x diploid crosses of potatoes. HortScience 25: 224-226.

Hijmans, R.J.; Garrett, K.A.; Huamán, Z.; Zhang, D.P.; Schreuder, M.. 2012. Assessing the geographic representativeness of genebank collections: the case of Bolivian wild potatoes. Conserv. Biol. 14: 1755-1765. 
Hijmans, R.J.; Spooner, D.M. 2001. Geographic distribution of wild potato species. Am. J. Bot. 88: 2101-2112.

Hijmans, R.J.; Spooner, D.M.; Salas, A.R.; Guarino, L.; de la Cruz, J., de la Cruz, J.. 2002. Atlas of Wild Potatoes. Int. Plant Genet. Res. Inst., Rome.

Huamán, Z., Hoekstra, R.; Bamberg, J.B.. 2000. The inter-genebank potato database and the dimensions of available wild potato germplasm. Am. J. Potato Res. 77: 353-362.

Jackson, M.T. 1990. Vavilov's Law of Homologous Series - Is it relevant to potatoes? Biol. J. Linn. Soc. 39: 17-25.

Jackson, S.A.; Hanneman Jr., R.E. 1999. Crossability between cultivated and wild tuber- and non-tuber-bearing Solanums. Euphytica 109: 51-67.

Jansky, S.H.; Jin, L.P.; Xie, K.Y.; Xie, C.H.; Spooner, D.M.. 2009a. Potato production and breeding in China. Potato Res. 52: 57-65.

Jansky, S.H.; Rouse, D.I.. 2003. Multiple disease resistance in interspecific hybrids of potato. Plant Dis. 87: 266-272.

Jansky, S.H.; Simon, R.; Spooner, D.M.. 2006. A test of taxonomic predictivity: Resistance to white mold in wild relatives of cultivated potato. Crop Sci. 46: 2561-2570.

Jansky, S.H.; Simon, R.; Spooner, D.M. 2008. A test of taxonomic predictivity: Resistance to early blight in wild relatives of cultivated potato. Phytopathology 98: 680-687.

Jansky, S.; Simon, R.; Spooner, D.. 2009b. A test of taxonomic predictivity: Resistance to the Colorado potato beetle in wild relatives of cultivated potato. J. Econ. Entomol. 102: 422-431.

Jarvis, A.; Lane, A.; Hijmans, R.J. 2008. The effect of climate change on crop wild relatives. Agr. Ecosyst. Environ. 126: 13-23.

Johnston, S.A.; den Nijs, T.P.M.; Peloquin, S.J.; Hanneman Jr., R.E. 1980. The significance of genic balance to endosperm development in interspecific crosses. Theor. Appl. Genet. 57: 5-9. 
Llontop, J.A.; Scurrah, M.M.; Franco, J.. 1989. Maria Huanca: New potato cultivar resistant to the potato cyst nematode (Globodera pallida). Revista ALAP 2: 77-89.

Love, S.L.; Pavek, J.J.; Thompson-Johns, A.; Bohl, W.. 1998. Breeding progress for potato chip quality in North American cultivars. Amer. J. Potato Res. 75: 27-36.

Masuelli, R.W., Camadro, E.L.; Erazzú, L.E.; Bedogni, M.C.; Marfil, C.F. 2009. Homoploid hybridization in the origin and evolution of wild diploid potato species. Plant Syst. Evol. 277: 143-151.

Maxted, N.; Dulloo, E.; Ford-Lloyd, B. V.; Iriondo, J.M.; Jarvis, A.. 2008. Gap analysis: A tool for complementary genetic conservation assessment. Diversity and Distributions 14: 1018-1030.

Maxted, N.; Kell, S.; Ford-Lloyd, B.; Dulloo, E.; Toledo, Á. 2012. Toward the systematic conservation of global crop wild relative diversity. Crop Sci. 52: 774-785.

McCouch, S.R.; McNally, K.L.; Wang, W.; Sackville Hamilton, R.2012. Genomics of gene banks: A case study in rice. Am. J. Bot. 99: 407-423.

Mendoza, A.O.; Haynes; F.L. 1974. Genetic relationship among potato cultivars grown in the United States. HortScience 9: 328-330.

Miller, J.K., Herman, E.M.; Jahn, M.; Bradford, K.J. 2010. Strategic research, education and policy goals for seed science and crop improvement. Plant Sci. 179: 645-652.

Mujeeb-Kazi, A.; Gul, A.; Farooq, M.; Rizwan, S.; Ahmad, I.. 2008. Rebirth of synthetic hexaploids with global implications for wheat improvement. Aust. J.Agr. Res.59: 391-398.

Navarre, D. A.; Pillai, S.S.; Shakya, R.; Holden, M.J.. 2011. HPLC profiling of phenolics in diverse potato genotypes. Food Chem. 127: 34-41.

Ochoa, C.M. 1990. The potatoes of South America. Cambridge University Press, Cambridge, USA. 
Ovchinnikova, A.; Krylova, E.; Gavrilenko, T.; Smekalova, T.; Zhuk, M.; Knapp, S.; Spooner, D.M. 2011. Taxonomy of cultivated potatoes (Solanum section Petota: Solanaceae). Bot. J. Linn. Soc. 165: 107-155.

Plaisted, R.L.; Hoopes, R.W. 1989. The past record and future prospects for the use of exotic potato germplasm. Am. J. Potato Res. 66: 603-627.

Ramírez-Villegas, J.; Khoury, C.; Jarvis, A.; Debouck, D.G.; Guarino, L.. 2010. A gap analysis methodology for collecting crop genepools: a case study with Phaseolus beans. PloS one 5: e13497.

Rieman, G.H.; Cooper, D.C.; Hougas, R.W. 1954. Potato varieties from species hybrids. Am. Potato J. 31: 1-11.

Ross, H. 1966. The use of wild Solanum species in German potato breeding of the past and today. Am. J. Potato Res. 43: 63-80.

Ross, H. 1979. Wild species and primitive cultivars as ancestors of potato varieties. p. 347. En: Zeven, A.C., Harten, A.M. van (eds.), Conference on Broadening the Genetic Base of Crops. Pudoc, Centre for Agricultural Publishing and Documentation, Wageningen, The Netherlands.

Rudorf, W. 1958. The significance of wild species for potato breeding. Potato Res. 1: 10-20.

Schafleitner, R.; Ramiíez, J., Jarvis, A.; Evers, D.; Gutiérrez, R., Scurrah, M. 2011. Adaptation of the potato crop to changing climates. p. 287-297. En: Crop Adaptation to Climate Change. Wiley-Blackwell, UK.

Scholes, R.J.; Walters, M.; Turak, E.; Saarenmaa, H.; Heip, C.H.; Tuama, É.Ó.; Faith, D.P.; Mooney, H. A.; Ferrier, S.; Jongman, R.H.; Harrison, I.J.; Yahara, T.; Pereira, H.M; Larigauderie, A.; Geller, G. 2012. Building a global observing system for biodiversity. Curr. Opin. Environ. Sustain. 4: 139-146.

Scott, G.J.; Suárez, V. 2012. Limits to growth or growth to the limits? Trends and projections for potatoes in China and their implications for industry. Potato Res. 55: 135-156. 
Scurrah, M.; Celis-Gamboa, C.; Chumbiauca, S.; Salas, A.; Visser, R.G.F. 2008. Hybridization between wild and cultivated potato species in the Peruvian Andes and biosafety implications for deployment of GM potatoes. Euphytica 164: 881-892.

Shrestha, R.; Arnaud, E.; Mauleon, R.; Senger, M.; Davenport, G..; Hancock, D.; Morrison, N.; Bruskiewich, R.; McLaren, G. 2010. Multifunctional crop trait ontology for breeders' data: field book, annotation, data discovery and semantic enrichment of the literature. AoB plants: plq008.

Spooner, D.M. 2009. DNA barcoding will frequently fail in complicated groups: An example in wild potatoes. Am. J. Bot. 96: 1177-1189.

Spooner, D.M.; Hijmans, R.J. 2001. Potato systematics and germplasm collecting, 1989-2000. Am. J. Potato Res. 78: 237-268.

Spooner, D.M.; Jansky, S.H.; Simon, R. 2009. Tests of taxonomic and biogeographic predictivity: Resistance to disease and insect pests in wild relatives of cultivated potato. Crop Sci. 49: 1367-1376.

Spooner, D.M., McLean, K.; Ramsay, G.; Waugh, R.; Bryan, G.J. 2005. A single domestication for potato based on multilocus amplified fragment length polymorphism genotyping. Proceed. Nat. Acad.Sci. USA 102: 14694-14699.

Spooner, D.M.; Núñez, J.; Trujillo, G.; Herrera, M.D.R.; Guzmán, F.; Ghislain, M. 2007. Extensive simple sequence repeat genotyping of potato landraces supports a major reevaluation of their gene pool structure and classification. Proceed. Nat. Acad. Sci. USA 104: 19398-19403.

Spooner, D.M.; Salas, A. 2006. Structure, biosystematics, and germplasm resources. p. 1-39. En: Gopal, J. (ed.), Handbook of Potato Production, Improvement, and Post-Harvest Management. Haworth's Press, Inc, Binghampton, New York.

Vavilov, N.I. 1922. The law of homologous series in variation. J. Genet. 12: 47-89.

Wang, F. 2011. Genetic diversity of the selected 64 potato germplasms revealed by AFLP markers. Mol. Plant Breed. 2: 22-29. 
Weber, B.; Hamernik, A.; Jansk, S. 2012. Hybridization barriers between Solanum tuberosum and wild Solanum raphanifolium. Genet. Resour. Crop Evol. 59: 1287-1293.

Zimnoch-Guzowska, E.; Sieczka, M.T.; Świeżyński, K.M. 1998. Development of parental lines for breeding potatoes resistant to viruses. p. 217-224. En: Proc. Intern. Symp.: Breed. Res. on Potatoes. Gro $\beta$ Lüsewicz, Germany.

Zitnak, A.; Johnston, G. 1970. Glycoalkaloid content of B5141-6 potatoes. Am. Potato J. 47: 256-260. 
